\title{
Possibilidades de valorização do coco pela aplicação de técnicas de joalheria
}

\author{
Mara Guerra 1; \\ Flávio Nascimento 2; \\ Raquel Canaan ${ }^{3}$;
}

resumo:

O coco é um material versátil e de ocorrência em quase todos os biomas brasileiros. Em diversos locais, é usado como matéria prima principal na confecção de objetos utilitários e decorativos, e é possível afirmar que dele se aproveita tudo, desde a casca até a água que se encontra em seu interior. O coco é usado na culinária, na confecção de fibras e possibilita outros usos, criando valor em toda a cadeia produtiva, pois os insumos derivados de sua estrutura podem ser aproveitados.

Como material na arte e através dos tempos, foi trabalhado por diversas culturas, se mostrando eficiente principalmente em suas características físicas. Existem registros de seu uso em datas e tempos diferentes pela aplicação de técnicas de escultura e lapidação, além de sua adornagem com ouro em folhas, esculturas, torneamento, estamparia. Este artigo busca apresentar uma pesquisa relacionada a este material, bem como possibilidades encontradas como resultado da mesma, da valorização desta matéria prima pela aplicação de técnicas de joalheria. Como resultado, é importante pontuar o aproveitamento do material disponível em grande quantidade em todo o território brasileiro e o resgate de técnicas consideradas patrimônio imaterial brasileiro e que estão se perdendo com o passar dos anos.

palavras-chave:

design; território; valorização; coco; joalheria.

Espaço reservado para organização do congresso.

\footnotetext{
${ }^{1}$ http://lattes.cnpq.br/2242061615883219

${ }^{2}$ http://lattes.cnpq.br/3202534145944006

${ }^{3}$ http://lattes.cnpq.br/3386924237539615
} 


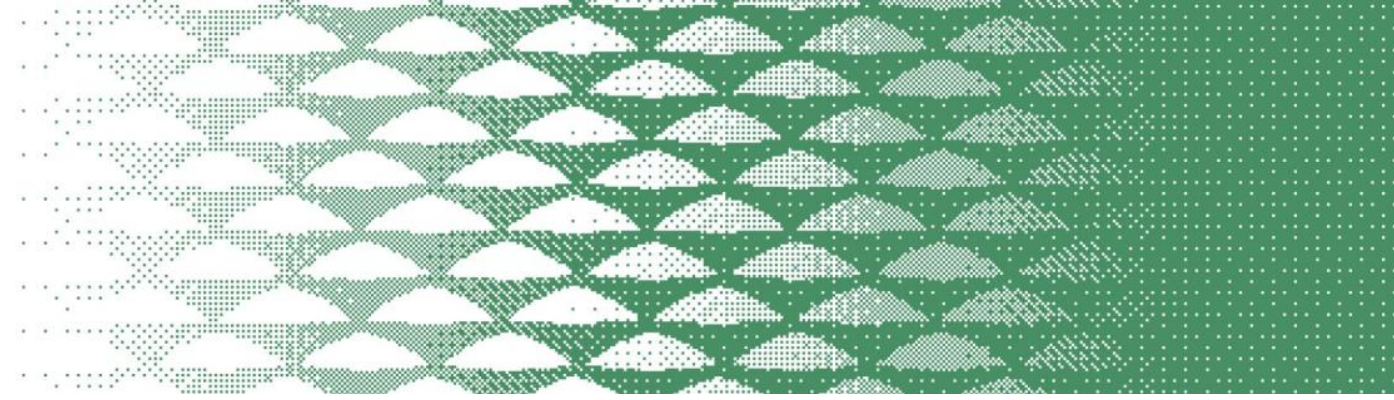

\section{Introdução}

Existe hoje no Brasil uma tendência, no setor de adornos, de valorização de artefatos relacionados a culturas locais e materiais da região, como o uso de sementes e folhas de plantas, as chamadas biojoias. De acordo com Benatti (2013, p. 33), essas joias são “[...] qualquer acessório de moda como colares, brincos, pulseiras, entre outros, produzidos a partir de matéria-prima natural como sementes, fibras de coco, madeira, etc." O coco, matéria prima da joalheria feita em Diamantina, é um material com grande potencial de aproveitamento, dentro do cenário exposto, por ser um produto que pode ser aproveitado por completo.

O Brasil é um país rico no que tange à cultura e à biodiversidade, que refletem nas possibilidades de desenvolvimento de conhecimentos contribuindo para valorizar os territórios pela criação de produtos ligados à sustentabilidade em diferentes níveis, mas que também podem convergir para as demandas dos consumidores por esse tipo de produtos.

A joalheria desenvolvida a partir do século XIX, principalmente no município de Diamantina/MG, criou importantes técnicas que qualificaram as joias produzidas e necessitam ser resgatadas e documentadas para que seus métodos construtivos não se percam com o passar do tempo. Por outro lado, o resgate das técnicas tradicionais contribuem para a promoção da sustentabilidade.

O coco é um material vegetal abundante na região do cerrado mineiro, sendo de fácil exploração e manejo e de grande durabilidade. Apesar da sua dureza, possibilita a criação das mais variadas formas e combinações, devido à facilidade de corte (trabalho de escultura ou torno com ferramentas específicas) e inserção de metais e pedras.

Este artigo apresenta a técnica tradicional de joalheria em coco e ouro desenvolvida no município de Diamantina, e tem como foco a sua inovação e aplicação a produtos sintonizados às demandas mais contemporâneas do setor joalheiro. Produzidas na região de Diamantina, desde meados do século XIX, as joias de coco e ouro sofreram poucas alterações ao longo do tempo. Pode-se dizer que o uso em conjunto do coco e do ouro representou, na época, uma inovação que possibilitou o desenvolvimento de peças de ourivesaria com estilo peculiar, que acabaram caindo no gosto popular e contribuindo para sua permanência no mercado joalheiro regional, até os dias atuais.

A joalheria brasileira tem hoje lugar de destaque no panorama mundial e é valorizada por fatores de identidade multicultural e de design, aliados aos materiais gemológicos e naturais abundantes em todo o território nacional. A identidade da joia brasileira pode ser fortalecida pela valorização não só de materiais nobres e raros, como também pela diversidade da combinação destes com outras habilidades e saberes, materiais e técnicas, além do desenvolvimento de capacidades tecnológicas próprias.

Além disso, o registro da técnica tradicional junto às novas possibilidades identificadas, colocam-se como uma oportunidade para a disseminação do ofício e da técnica como saber local e contribuir para a valorização de modos de produção e atividades econômicas importantes na história regional, como patrimônio cultural. O design aplicado à técnica produtiva do coco e ouro se apresenta como uma perspectiva de inovação de uso e de resposta às demandas atuais na área de joalheria às condições locais de coleta e manejo sustentáveis da matéria prima.

\section{Design e possibilidades para valorização de produtos do território}

Nos territórios nascem e se desenvolvem os valores que, com o tempo, consolidam patrimônios imateriais. Comunicar valores imateriais de um território é essencial para a manutenção da produção característica de um lugar. De acordo com Burke (2007), todo país tem várias tradições, porém no Brasil elas são bem visíveis; característica da hibridização de múltiplas heranças e tradições culturais. Neste trabalho, o conceito de tradição é útil porque levanta a questão da autenticidade, própria de uma tradição, 


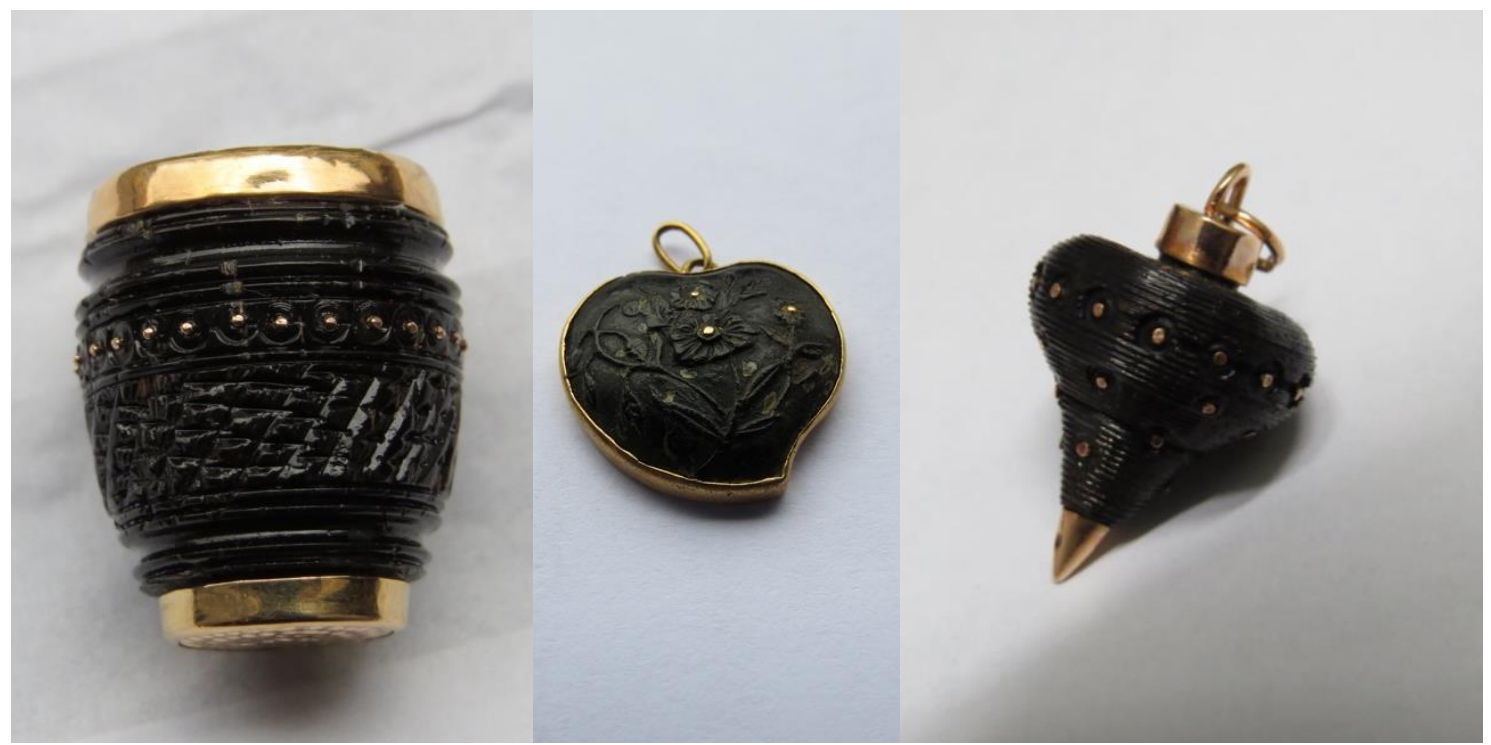

FIGURA 01 - Dedal, pingente coração, berloque peão

Fonte: Joalheria Chica da Silva. Foto: Samia Abbas

A variação de detalhes entre o trabalho de cada oficina é quase imperceptível para a maioria das pessoas, está em alguns detalhes na combinação de técnicas de filigrana e no entalhe do coco. Alguns pontos podem ser destacados no sentido tanto do resgate da técnica como do uso do coco como matéria prima para a joalheria. Entrevistas realizadas ao longo da pesquisa realizada, apontam a ourivesaria em Diamantina como herança portuguesa, trazida por ourives portugueses que se instalaram na região e ensinaram a técnica a aprendizes em suas oficinas, e estes continuaram desenvolvendo a prática na região.

Todo o processo da joalheria em coco e ouro apoia-se em uma tecnologia primária, e o ferramental utilizado consiste basicamente nos da joalheria artesanal tradicional, sendo que algumas ferramentas são adaptadas pelos próprios artesãos. As ferramentas específicas utilizadas são as mesmas da técnica da estamparia, que alguns produzem e outros têm ferramenteiros que fabricam para as oficinas. Quanto à permanência da técnica na região percebe-se que ela está condicionada à entrada de novos interessados no seu aprendizado com os artesãos que a praticam.

As tipologias dos produtos também se restringem a anéis, pingentes, cruzes e brincos e grande parte dos artesãos aponta não ter criado novos modelos por falta de ideia para a criação. Nesse ponto, encontramos oportunidades significativas no sentido da capacitação de pessoas para o aprendizado da técnica, bem como da inovação do produto no sentido de atingir novos públicos e mercados.

Pelas particularidades intrínsecas à técnica produtiva, a joalheria em coco e ouro pode ser considerada um bem cultural. As particularidades formais e materiais que diferem esta joalheria de outras produzidas na região e fora dela, já a identificam como produto local. Porém a técnica do coco e ouro apresenta possibilidades de inovação a fim de atender novas demandas e mercados. Uma inovação técnica passível de aplicação está relacionada ao processo de escultura das cascas. A aplicação de outras técnicas como as de lapidação sobre o coco permite melhor aproveitamento das estruturas dos seus tegumentos, e ainda criar diferentes superfícies, conferindo um novo padrão estético formal aos produtos.

O Quadro desenvolvido a partir das observações de pesquisa, sintetiza a joalheria em coco e ouro, apresentando as contas tradicionais em coco da Bahia e as técnicas utilizadas em cada uma das 

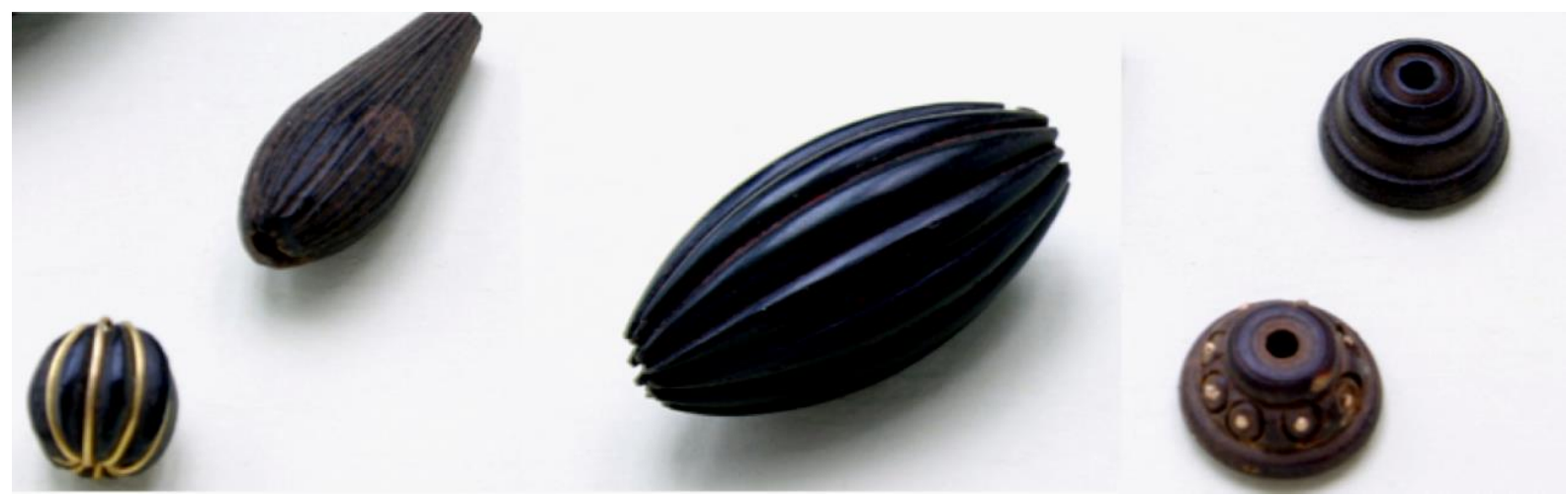

FIGURA 01 - Contas tradicionais de coco e ouro.

Fonte: Foto de Antônio Mattos, 2014.

O design de novas peças em coco e ouro pode ser incorporado às produzidas atualmente, criando novas opções para o mercado atual e demandas atuais. Como proposta de inovação material está o aproveitamento dos cocos, principalmente Indaiá e Macaúba. Nesses cocos foram realizados testes com equipamento de lapidação, com a finalidade de otimizar o formato da estrutura dos mesmos, possibilitando o desenvolvimento de modelos diferentes e maiores que os tradicionais. A Figura a seguir mostra possibilidades de trabalho na forma do coco, podendo ser facetado ou esculpido com o rebolo usado na lapidação de gemas; foi observado que o equipamento de rebolo em carbeto de silício responde bem para o uso nos cocos, não apresentando dificuldades na execução da forma e nem acarretando o entupimento do mesmo.

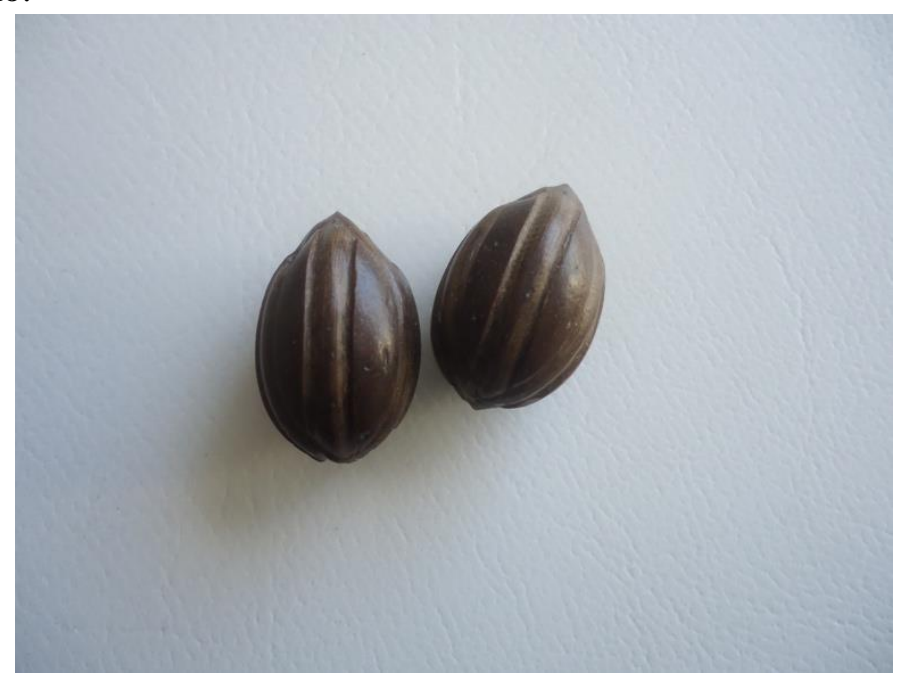

FIGURA 03 - Coco trabalhado em equipamento de lapidação e com fresas. Fonte: Marcus Cattapreta, 2013.

Para aplicar a técnica de lapidação nos cocos, foram feitos três modelos de contas nos cocos macaúba e indaiá. O resultado da aplicação da técnica de lapidação no coco foi considerada viável por apresentar bom polimento e facilidade de corte usando os equipamentos de lapidação. 
e reconhecimento de origem. Essa contribuição pode ser alcançada por meio da inovação da técnica e ativação de valores do território.

A inovação aliada a tradição e cultura da joalheria em Diamantina pode representar um novo modelo de joalheria no Brasil, que valoriza, através da inserção do design e valorização de materiais disponíveis localmente como o coco. O estudo da cadeia de valor da joalheria em coco e ouro representa uma oportunidade de desenvolvimento social, econômico, cultural e ambiental por meio da inserção de design nos processos do beneficiamento dos cocos nativos da região e da comunicação desse tipo de produção no local.

Possibilities of valuing coconut through the application of jewelry techniques

Abstract: Coconut is a versatile material that occurs in almost all Brazilian biomes. In several places, it is used as the main raw material in the manufacture of utilitarian and decorative objects, and it is possible to say that everything is used from it, from the bark to the water inside. Coconut is used in cooking, in the manufacture of fibers and allows for other uses, creating value throughout the production chain, as the inputs derived from its structure can be used. As a material in art and through the ages, it was worked by different cultures, showing itself to be efficient mainly in its physical characteristics. There are records of its use at different dates and times through the application of sculpting and cutting techniques, in addition to its adornment with gold leaves, sculptures, turning, stamping. This article seeks to present a research related to this material, as well as possibilities found as a result of it, of the valorization of this raw material through the application of jewelry techniques. As a result, it is important to point out the use of the material available in large quantities throughout the Brazilian territory and the rescue of techniques considered Brazilian intangible heritage and that are being lost over the years.

Keywords: design; territory; valorization; coconut; jewelry.

\title{
Referências bibliográficas
}

ARANTES. Antônio. $\mathbf{O}$ patrimônio imaterial e a sustentabilidade de sua salvaguarda. Resgate, v. 3, 2004.

\author{
ARISTONE, Flávio. Exploração auto-sustentável da bocaiúva no Pantanal Sul-Mato- \\ Gossense: Geração de renda e equilibrio do meio-ambiente. Universidade Federal do Mato \\ Grosso do Sul-UFMS. Centro Nacional de Pesquisa e Desenvolvimento Tecnológico-CNPq, 18 p.2006. \\ Disponível em<http://www.entabrasil.com.br>
}

BENATTI, Lia Paletta. Biojoia. In: Biojoia: design e inovação aplicados às sementes brasileiras. Organização Lia Paletta Benatti. Belo Horizonte: Gabbo Design,2013.

BURKE, Peter. Percursos e desafios da pesquisa e do ensino de história da educação. Uberlândia: EDUFU, 2007.

CAMPOS, Ana Paula de. Arte-Joalheria: uma cartografia pessoal. Campos/ Campinas, SP: [s.n.], 2011. 
CANAAN, R. P. Gemas e joias: a gestão pelo design aplicada à cadeia de valor de arranjos produtivos locais. 2013. 117 f. Dissertação (Mestrado) - Escola de Design, Programa de Pós-Graduação em Design da Universidade do Estado de Minas Gerais, Belo Horizonte, 2013.

CAVALCANTI, Virgínia P.; ANDRADE, Ana Maria; SILVA, Germannya A. A comunicação da sustentabilidade de produtos e serviços. In: Cadernos de Estudos Avançados em Design Sustentabilidade I. Barbacena, MG: EdUEMG, 2009.

FAVARO, Henny Aguiar Bizarro Rosa. Design de Joias e pesquisa acadêmica: limites e sobreposições.2014. 288 f. Tese de Doutorado em Arquitetura e Urbanismo. Universidade Presbiteriana Makenzie, São Paulo, 2013.

FACTUM, A. B. S. Joalheria escrava baiana: a construção histórica do design de joias brasileiro. 2009. Tese (Doutorado em Arquitetura e Urbanismo) - Faculdade de Arquitetura e Urbanismo da Universidade de São Paulo. São Paulo, 2009.

FRANCHESCHI, Humberto M. O. Ofício da prata no Brasil. Rio de Janeiro: Studio HMF, 1988.

FURTADO, Júnia Ferreira. 0 Livro da capa verde: o regimento Diamantino de 1771 e a vida no distrito diamantino no período da Real Extração. São Paulo: Annablume; Belo Horizonte: PPGH/UFMG, 2008.

KRUCKEN, Lia; TRUSEN, Christoph. A comunicação da Sustentabilidade de Produtos e Serviços. In: Cadernos de estudos avançados em design: design e sustentabilidade I. Organização Dijon de Moraes e Lia Krucken, 2. ed. Barbacena, MG: EdUEMG, 2013.

KRUCKEN, Lia. Design e território: valorização de identidades. São Paulo: Studio Nobel, 2009.

LAGES, Vinícius; BRAGA, Christiano; MORELLI, Gustavo (Orgs.). Territórios em movimento: cultura e identidade como estratégia de inserção competitiva. Rio de Janeiro: Relume Dumará / Brasília, DF: SEBRAE, 2004.

MOTA, Rosa Maria dos Santos. Glossário do uso do ouro no norte de Portugal. Porto: UCE, 2011. NEVES, Cláudia Costa; REZENDE, Sonaly. Aspectos Sócio-demográficos do Município de Diamantina na transição da atividade mineradora para as atividades turística e educacional. XII Seminário sobre Economia Mineira[ Proceedingsofthe 12 thseminarontheEconomyof Minas Gerais, 2006 fromCedeplar, Universidade Federal de Mina Gerais]. Belo Horizonte, 2006.

OZANAN, Luiz H. A JOIA MAIS PRECIOSA DO BRASIL: joalheria em Minas Gerais, 1735 1815. Tese de doutorado. Universidade Federal de Minas Gerais, 2013.

ROCHA, Silvia C. S.; ROCHA Paulo R. O Brasil da joia, design e arte. São Paulo: Ed. Do Autor, 2014.

SACHS, Ignacy. Caminhos para o desenvolvimento sustentável. Rio de Janeiro: Garamond, 2009.

SANTOS, Joaquim Felício dos. Memórias do distrito diamantino. Belo Horizonte: Itatiaia; São Paulo: Ed. Universidade de São Paulo,1976.

SILVEIRA, Carlos Eduardo et al. Caminhos do Turismo em Diamantina: a relação com a origem mineradora, a cultura e o título de patrimônio cultural da humanidade. Revista Vozes dos Vales da UFVJM: Publicações acadêmicas- MG- Brasil - no 01- Ano I. 05/2012. Reg. 120.2.095PROEXC/UFVJM. Disponível em www.ufvjm.edu/vozes. 